\title{
RESPOSTA DO MILHO A DIFERENTES DOSAGENS DE NITROGÊNIO E FÓSFORO APLICADOS AO SOLO ${ }^{1}$
}

\author{
Lúcia de Fátima Castro Lucena ${ }^{2}$, Francisco Assis de Oliveira ${ }^{3}$, Ivandro de França da Silva ${ }^{4}$ \& \\ Alberício Pereira de Andrade ${ }^{4}$
}

\begin{abstract}
RESUMO
O estudo foi conduzido no ano agrícola de 1996, em condições de sequeiro, em um Latossolo Vermelho-Amarelo distrófico (LV) no município de Areia, PB, com o objetivo de se avaliar os efeitos das dosagens de nitrogênio $\left(40,80,120\right.$ e $\left.160 \mathrm{~kg} \mathrm{ha}^{-1} \mathrm{de} \mathrm{N}\right)$ e de fósforo $\left(60,120,180\right.$ e $240 \mathrm{~kg} \mathrm{ha}^{-1} \mathrm{de}^{-}$ $\mathrm{P}_{2} \mathrm{O}_{5}$ ) sobre algumas características da cultura do milho, cultivar BR 5033. Utilizou-se o delineamento experimental de blocos ao acaso, em esquema fatorial $4^{2}+2$ tratamentos adicionais (zero de $\mathrm{N}$ e zero de $\mathrm{P}_{2} \mathrm{O}_{5}$ ) com três repetições. Foram avaliados os dados referentes às variáveis: diâmetro caulinar, número de grãos/espiga, peso de espiga com e sem palha, e rendimento de grãos pela cultura. $\mathrm{O}$ rendimento da cultura respondeu positivamente ao $\mathrm{N} \mathrm{e}_{2} \mathrm{O}_{5}$, até as dosagens de $111,1 \mathrm{~kg} \mathrm{ha}^{-1} \mathrm{e}$ $197,6 \mathrm{~kg} \mathrm{ha}^{-1}$, respectivamente.
\end{abstract}

Palavras-chave: Zea mays, rendimento, adubação

\section{RESPONSE OF CORN TO DIFFERENT DOSES OF NITROGEN AND PHOSPHORUS APPLIED TO SOIL}

\begin{abstract}
A study was carried out during 1996, in Yellow Red Latosol at Areia in the state of Paraíba (Brazil). The objective of this study was to evaluate the effects of increasing doses of nitrogen $(40,80,120$, and $160 \mathrm{~kg} \mathrm{ha}^{-1}$ of $\mathrm{N}$ ) and phosphorus $\left(60,120,180\right.$, and $240 \mathrm{~kg} \mathrm{ha}^{-1}$ of $\left.\mathrm{P}_{2} \mathrm{O}_{5}\right)$ and two additional treatments $\left(0 \mathrm{~kg} \mathrm{ha}^{-1}\right.$ of $\mathrm{N}$ and $0 \mathrm{~kg} \mathrm{ha}^{-1}$ of $\left.\mathrm{P}_{2} \mathrm{O}_{5}\right)$ on some characteristics of corn crop, cv. BR 5033, under rainfed conditions, in a sandy clay loam soil. The experimental design was of randomized blocks in a factorial scheme $4^{2}+2$ ( 4 levels $\times 2$ factors +2 additional treatments - level 0 of $\mathrm{N}$ and $\mathrm{P}_{2} \mathrm{O}_{5}$ ) with three replications. Plant parameters evaluated were stem diameter, number of grains per cob, cob weight with and without straw, and grain yield. Grain yield showed a response to the application of $\mathrm{N}$ and $\mathrm{P}_{2} \mathrm{O}_{5}$ up to levels of $111.1 \mathrm{~kg} \mathrm{ha}^{-1}$ and $197.6 \mathrm{~kg} \mathrm{ha}^{-1}$, respectively.
\end{abstract}

Key words: Zea mays, grain yield, fertilization

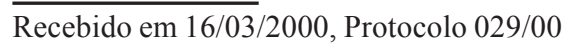

${ }^{1}$ Extraído da Dissertação de Mestrado em Manejo de Solo e Água, apresentada pelo primeiro autor ao Centro de Ciências Agrárias da UFPB, Areia, PB

${ }^{2}$ Eng. Química, M.Sc., Sec. Agricultura, Irrigação e Abastecimento da Paraíba - SAIA, Praça. Clementino Procópio 97. CEP 58101 - 000, Campina Grande, PB

${ }^{3}$ Eng. Agr., Dr., Prof. Adj., Dep. De Solos e Eng. Rural, Centro de Ciências Agrárias, UFPB, CEP 58397 - 000 , Areia, PB. Bolsista do CNPq. Fone: (0xx83) 322 2457, Fax: (0xx83) 362 2259. E-mail: oliveira@cca.ufpb.br

${ }^{4}$ Professor Adjunto, Doutor, DSER/CCA/UFPB, CEP 58397 - 000, Areia, PB. Fone: (0xx83) 362 2300, Fax: (0xx83) 3622259. E-mail: ivandro@cca.ufpb.br 


\section{INTRODUÇÃO}

No contexto da agricultura brasileira, o cultivo do milho representa importante papel socioeconômico, principalmente na alimentação das populações de baixa renda, cujo consumo atinge a ordem de $33 \mathrm{~kg}$ per capita por ano (Viola, 1980). $\mathrm{Na}$ região Nordeste, a área cultivada com milho é de aproximadamente três milhões de hectares, com produtividade média de $614 \mathrm{~kg} \mathrm{ha}^{-1}$ (Carvalho et al., 1997). Vários fatores, dentre os quais a irregularidade pluvial, os sistemas tradicionais de cultivo, o manejo cultural e a ausência de uma adubação balanceada, principalmente a nitrogenada e a fosfatada, concorrem para o insucesso agrícola na região. Esses fatores, associados ao uso e ao manejo do solo, contribuem para que haja resposta do milho à adubação nitrogenada (Muzilli et al., 1989). Segundo França et al. (1986) o nitrogênio e o fósforo são os dois nutrientes, em condições naturais, que mais limitam a produção de grãos no Brasil, especialmente a das gramíneas. Resultados de experimentos conduzidos sob diversas condições de solo, clima e sistemas de cultivo, em Minas Gerais (França et al., 1986) e em São Paulo (Cantarella \& Raij, 1986) mostraram que, em geral, de 70 a $90 \%$ dos ensaios com a cultura do milho responderam à aplicação de N. Através de experimentos em condições de campo, Vieira et al. (1976) e Melgar et al. (1991) constataram que o rendimento de grãos de milho aumentou linearmente em função das dosagens de N (40, 80 e $\left.120 \mathrm{~kg} \mathrm{ha}^{-1}\right)$ aplicadas ao solo, em várias formas de parcelamento. Ao lado do nitrogênio a deficiência do fósforo limita a produtividade do milho, cuja cultura é mais exigente a fósforo por ocasião da formação e desenvolvimento dos grãos, contribuindo para a formação de espigas mal-formadas, tortas e com falhas, maturação retardada e desuniforme. Fornasiere Filho (1992) reverencia efeito positivo da aplicação do fósforo a lanço nas dosagens de 160 e $320 \mathrm{~kg} \mathrm{ha}^{-1}$ de $\mathrm{P}_{2} \mathrm{O}_{5}$ na produção de grãos e de palha de milho, porém Muzilli (1982) encontrou, para o Estado do Paraná resposta do milho ao fósforo até a dosagem de $120 \mathrm{~kg} \mathrm{ha}^{-1}$ de $\mathrm{P}_{2} \mathrm{O}_{5}$ aplicada a lanço.

O trabalho objetivou estudar, em condições de campo e de precipitação natural, o efeito de níveis de adubação nitrogenada e fosfatada, isoladas e/ou associadas sobre o desenvolvimento e rendimento da cultura do milho (Zea mays L.) cultivar BR 5033.

\section{MATERIAL E MÉTODOS}

Em 1996, no município de Areia, microrregião do Brejo paraibano, o ensaio foi conduzido em um Latossolo VermelhoAmarelo distrófico, textura média (Brasil, 1972) com granulometria na camada superficial $0-20 \mathrm{~cm}$ de $502 \mathrm{~g} \mathrm{~kg}^{-1} \mathrm{de}$ areia total, $153 \mathrm{~g} \mathrm{~kg}^{-1}$ de silte e $345 \mathrm{~g} \mathrm{~kg}^{-1}$ de argila e, como característica química, apresenta $\mathrm{pH}$ em água $=5,20$, $\mathrm{Al}=10,8 \mathrm{mmol} \mathrm{dm}^{-3}, \mathrm{Ca}=27,0 \mathrm{mmol} \mathrm{dm}^{-3}, \mathrm{Mg}=9,5 \mathrm{mmol} \mathrm{dm}^{-3}$, $\mathrm{P}=1,8 \mathrm{mg} \mathrm{dm}^{-3}, \mathrm{~K}=31,2 \mathrm{mg} \mathrm{dm}^{-3}$ e $17,0 \mathrm{~g} \mathrm{dm}^{-3}$ de matéria orgânica. Com base no alumínio trocável fez-se a correção da acidez do solo 40 dias antes da semeadura, aplicando-se 3,5 t ha $^{-1}$ de calcário dolomítico com PRNT de $62 \%$. O corretivo foi distribuído na área após a aração e incorporado ao solo através de duas gradagens cruzadas. Como adubação fixa, aplicaram-se $60 \mathrm{~kg} \mathrm{ha}^{-1}$ de $\mathrm{K}_{2} \mathrm{O}$, na forma de cloreto de potássio. $\mathrm{O}$ local do experimento dista aproximadamente $2 \mathrm{~km}$ a Oeste do ponto de coordenadas geográficas $6^{\circ} 55^{\prime} 12^{\prime \prime}$ de latitude Sul, $35^{\circ} 42^{\prime} 15^{\prime \prime}$ de longitude Oeste de Greenwich e $575 \mathrm{~m}$ de altitude. Durante a condução do ensaio foram registrados $337,4 \mathrm{~mm}$ de chuva. O maior veranico, de 12 dias, ocorreu dos 20 aos 32 dias do ciclo da cultura.

Foram testados os níveis de $\mathrm{N}\left(0,40,80,120\right.$ e $160 \mathrm{~kg} \mathrm{ha}^{-1} \mathrm{de}$ N) e de $\mathrm{P}\left(0,60,120,180\right.$ e $\left.240 \mathrm{~kg} \mathrm{ha}^{-1} \mathrm{de}_{2} \mathrm{O}_{5}\right)$ tendo como fontes a uréia e o superfosfato triplo, respectivamente. $\mathrm{O}$ delineamento experimental utilizado foi o de blocos ao acaso, com os tratamentos distribuídos em arranjo fatorial de $4^{2}$ (4 níveis de $\mathrm{N}$ x 4 níveis de $\mathrm{P}_{2} \mathrm{O}_{5}$ ) mais dois tratamentos adicionais, zero de $\mathrm{N}\left(\mathrm{N}_{\mathrm{O}}\right)$ e zero de $\mathrm{P}_{2} \mathrm{O}_{5}\left(\mathrm{P}_{\mathrm{O}}\right)$ com três repetições. No tratamento $\mathrm{N}_{\mathrm{O}}$ foram aplicados $120 \mathrm{~kg} \mathrm{ha}^{-1} \mathrm{de}_{2} \mathrm{O}_{5} \mathrm{e}$, no $\mathrm{P}_{\mathrm{O}}, 80 \mathrm{~kg} \mathrm{ha}^{-1} \mathrm{de} \mathrm{N}$. Foram aplicados, também, $50 \%$ de $\mathrm{N}$ nos sulcos, por ocasião da semeadura, e os outros $50 \%$ em cobertura, aos 45 dias após a emergência (DAE), e o P foi aplicado a lanço, antes da semeadura e incorporado ao solo através de gradagem. A unidade experimental constou de uma área de $24 \mathrm{~m}^{2}(6 \mathrm{x} 4 \mathrm{~m})$ com área útil $8 \mathrm{~m}^{2}(4 \times 2 \mathrm{~m})$ da parte central da parcela. Para se testar o efeito dos tratamentos usou-se a cultura do milho (Zea mays L.) cultivar BR 5033. Em 25/4/96, procedeu-se à semeadura em sulcos espaçados 1,0 m entre fileiras e $0,2 \mathrm{~m}$ entre covas, e a colheita foi processada em 30/9/69. Aos 15 dias após a DAE procedeu-se ao desbaste deixando-se, nesta operação, cinco plantas por metro. Para o controle das ervas daninhas, foram realizadas três capinas manuais e aplicadas duas pulverizações com folidol, na dosagem de $0,8 \mathrm{~L} \mathrm{ha}^{-1}$, para combater a lagartado-cartucho (Spodoptera frugiperda).

Avaliaram-se os dados referentes às variáveis diâmetro caulinar, número de grãos por espiga, peso de espiga com e sem palha, altura de planta e rendimento de grãos. Os dados foram submetidos à análise de variância e as médias comparadas pelo teste de Tukey, a 5\% de probabilidade. Para as variáveis altura de planta e rendimento de grãos, foram determinadas as curvas de respostas através da análise de regressão polinomial. Para a análise de máxima eficiência física (MEF) procedeu-se segundo metodologia descrita em Braga (1992).

\section{RESULTADOS E DISCUSSÃO}

Os 337,4 mm de chuva registrados durante a condução do ensaio, certamente não foram suficientes para atender às reais necessidades da cultura; inclusive, durante o veranico de 12 dias na fase inicial do ciclo da cultura, foram observados sintomas visuais de murcha que, segundo Formasiere Filho (1992) podem ter concorrido para limitar o rendimento da cultura pois, nesse estádio de desenvolvimento, o milho necessita de maior quantidade de água no solo, à sua disposição.

De acordo com a análise de variância, ocorreu efeito significativo $(\mathrm{p} \leq 0,01)$ dos tratamentos de nitrogênio e fósforo sobre as variáveis analisadas, exceto para a altura de plantas,

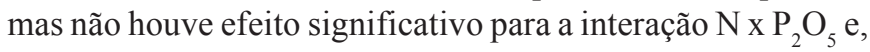
também, do fatorial versus tratamentos adicionais $\left(\mathrm{N}_{\mathrm{O}}\right)$ e $\left(\mathrm{P}_{\mathrm{O}}\right)$. Segundo Gamboa (1980) efeitos positivos da interação de N x $\mathrm{P}_{2} \mathrm{O}_{5}$ sobre a produção de grãos de milho ocorrem com mais freqüência quando a adubação nitrogenada é feita na forma amoniacal, proporcionando maiores incrementos na absorção de fósforo pela planta. 
A Tabela 1 apresenta os resultados obtidos em que, por meio da comparação entre as médias pelo teste de Tukey a 5\% de probabilidade, é possível se constatar que não houve efeito do nitrogênio sobre os resultados de altura de planta e de grãos por espiga, mas a dosagem de $40 \mathrm{~kg} \mathrm{ha}^{-1}$ de $\mathrm{N}$ reduziu de forma significativa $(p \leq 0,05)$ o peso de espiga com e sem palha. Idêntico comportamento ocorreu com o fósforo, cujas dosagens $\left(120,160\right.$ e $240 \mathrm{~kg} \mathrm{ha}^{-1}$ de $\left.\mathrm{P}_{2} \mathrm{O}_{5}\right)$ não apresentaram resultados diferentes mas superaram, de forma significativa, a dosagem de $60 \mathrm{~kg} \mathrm{ha}^{-1}$ de $\mathrm{P}_{2} \mathrm{O}_{5}$, no que se refere às variáveis grãos/espiga e peso de espiga com e sem palha.

Tabela 1. Valores médios ${ }^{1}$ de diâmetro caulinar, número de grãos por espiga, peso de espiga com e sem palha da cultivar de milho BR 5033, cultivado em diferentes níveis de nitrogênio e fósforo aplicados ao solo

\begin{tabular}{|c|c|c|c|c|}
\hline \multirow[b]{2}{*}{ Tratamento } & \multirow{2}{*}{$\begin{array}{c}\text { Diâmetro } \\
\text { Caulinar } \\
\mathrm{mm}\end{array}$} & \multirow{2}{*}{$\begin{array}{c}\text { Grãos } \\
\text { por Espiga } \\
n^{0}\end{array}$} & \multicolumn{2}{|c|}{ Peso da Espiga $\left(\mathrm{kg} \mathrm{ha}^{-1}\right)$} \\
\hline & & & $\begin{array}{l}\text { Com } \\
\text { Palha }\end{array}$ & $\begin{array}{c}\text { Sem } \\
\text { Palha }\end{array}$ \\
\hline \multicolumn{5}{|l|}{$\mathrm{N}\left(\mathrm{kg} \mathrm{ha}^{-1}\right)$} \\
\hline 40 & $13,5 \mathrm{a}$ & $295,2 \mathrm{a}$ & $2810,4 \mathrm{~b}$ & $2003,1 \mathrm{~b}$ \\
\hline 80 & $12,8 \mathrm{a}$ & $311,4 \mathrm{a}$ & 3564,6 a & $2567,7 \mathrm{a}$ \\
\hline 120 & $13,9 \mathrm{a}$ & $320,1 \mathrm{a}$ & $3575,0 \mathrm{a}$ & $2815,6 \mathrm{a}$ \\
\hline 160 & $13,7 \mathrm{a}$ & $316,6 \mathrm{a}$ & $3434,4 \mathrm{a}$ & $2481,2 \mathrm{a}$ \\
\hline \multicolumn{5}{|l|}{$\mathrm{P}_{2} \mathrm{O}_{5}\left(\mathrm{~kg} \mathrm{ha}^{-1}\right)$} \\
\hline 60 & $12,5 \mathrm{a}$ & $264,0 \mathrm{~b}$ & $2438,5 \mathrm{~b}$ & $1811,4 \mathrm{~b}$ \\
\hline 120 & $13,5 \mathrm{a}$ & $325,9 \mathrm{a}$ & $3506,2 \mathrm{a}$ & $2557,3 \mathrm{a}$ \\
\hline 180 & $13,9 \mathrm{a}$ & $337,2 \mathrm{a}$ & $3649,0 \mathrm{a}$ & $2692,7 \mathrm{a}$ \\
\hline 240 & $13,9 \mathrm{a}$ & $316,2 \mathrm{a}$ & $3790,6 \mathrm{a}$ & $2806,2 \mathrm{a}$ \\
\hline Fatorial & $13,5 \mathrm{a}$ & $310,8 \mathrm{a}$ & $3346,1 \mathrm{a}$ & 2466,9 a \\
\hline $0 \mathrm{~kg} \mathrm{ha}^{-1}$ de $\mathrm{N}$ & $11,2 \mathrm{~b}$ & $168,6 \mathrm{~b}$ & $1195,0 \mathrm{~b}$ & $720,0 \mathrm{~b}$ \\
\hline $0 \mathrm{~kg} \mathrm{ha}^{-1}$ de $\mathrm{P}_{2} \mathrm{O}_{5}$ & $11,4 \mathrm{~b}$ & $207,6 \mathrm{~b}$ & $1700,0 \mathrm{~b}$ & $1075,0 \mathrm{~b}$ \\
\hline
\end{tabular}

Ainda na Tabela 1, na qual se comparam os resultados médios do fatorial com os tratamentos adicionais, ou seja, $0 \mathrm{~kg} \mathrm{ha}^{-1}$ de $\mathrm{N}\left(\mathrm{N}_{\mathrm{O}}\right)$ e $0 \mathrm{~kg} \mathrm{ha}^{-1}$ de $\mathrm{P}_{2} \mathrm{O}_{5}\left(\mathrm{P}_{\mathrm{O}}\right)$ observa-se que os resultados do fatorial superaram significativamente $(\mathrm{p} \leq 0,01)$ os tratamentos adicionais sobre os resultados das variáveis analisadas não havendo, no entanto, diferença entre $\mathrm{N}_{0}$ e $\mathrm{P}_{\mathrm{O}}$. Os resultados médios do fatorial superaram os tratamentos $\mathrm{N}_{\mathrm{O}}$ e $\mathrm{P}_{\mathrm{O}}$ em $20,5 \%$ e $18,4 \%$, com relação ao diâmetro caulinar em $84,3 \%$ e $49,7 \%$, com relação ao número de grãos por espiga, em 180,0 e $96,8 \%$, o peso de espiga com palha e em 242,6 e $129,5 \%$, o peso de espiga sem palha, respectivamente.

Na Figura 1 são apresentados os resultados de altura de planta em função dos níveis de $\mathrm{N}$ aplicados ao solo. Verifica-se que os dados de altura de planta se ajustaram significativamente ( $\mathrm{p} \leq 0,01)$ a uma equação de regressão do $2^{\circ}$ grau, cujo coeficiente de determinação explica que o efeito dos tratamentos com $\mathrm{N}$ sobre a variável analisada foi da ordem de $93,7 \%$. A dosagem de máxima eficiência física (DMEF) foi de $100,0 \mathrm{~kg} \mathrm{ha}^{-1}$ de N para uma altura máxima de planta $(146,3 \mathrm{~cm})$.

Idêntico comportamento ocorreu com a altura de planta em função dos níveis de fósforo, no qual o efeito dos tratamentos foi explicado pelo coeficiente de determinação em 99,3\%, verificando-se que a altura máxima $(151,2 \mathrm{~cm})$ seria atingida com a aplicação de $177,3 \mathrm{~kg} \mathrm{ha}^{-1}$ de $\mathrm{P}_{2} \mathrm{O}_{5}$, como referencia a Figura 2.

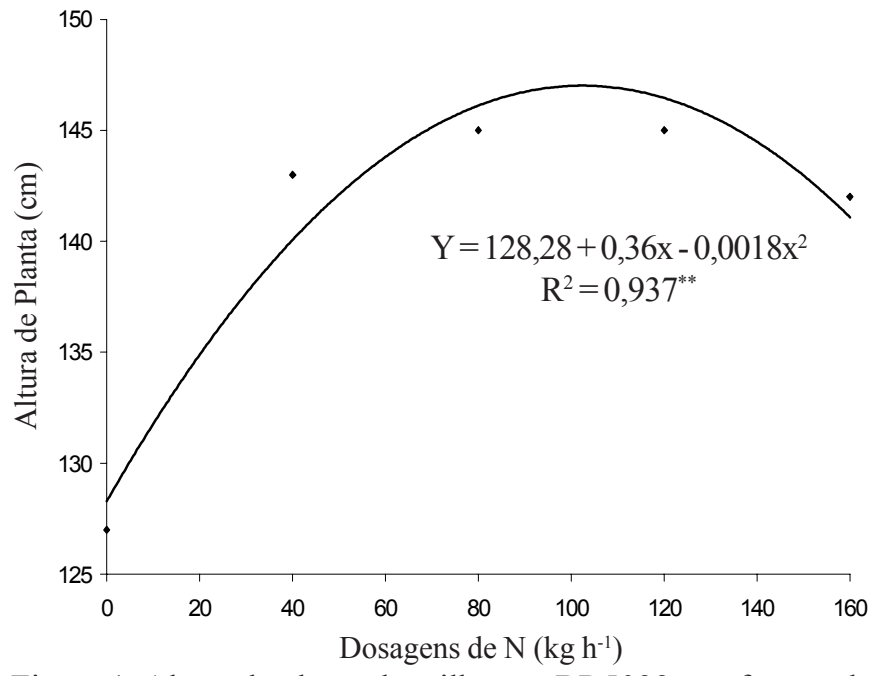

Figura 1. Altura de planta de milho, cv. BR5033, em função de dosagens crescentes de $\mathrm{N}$ aplicadas ao solo

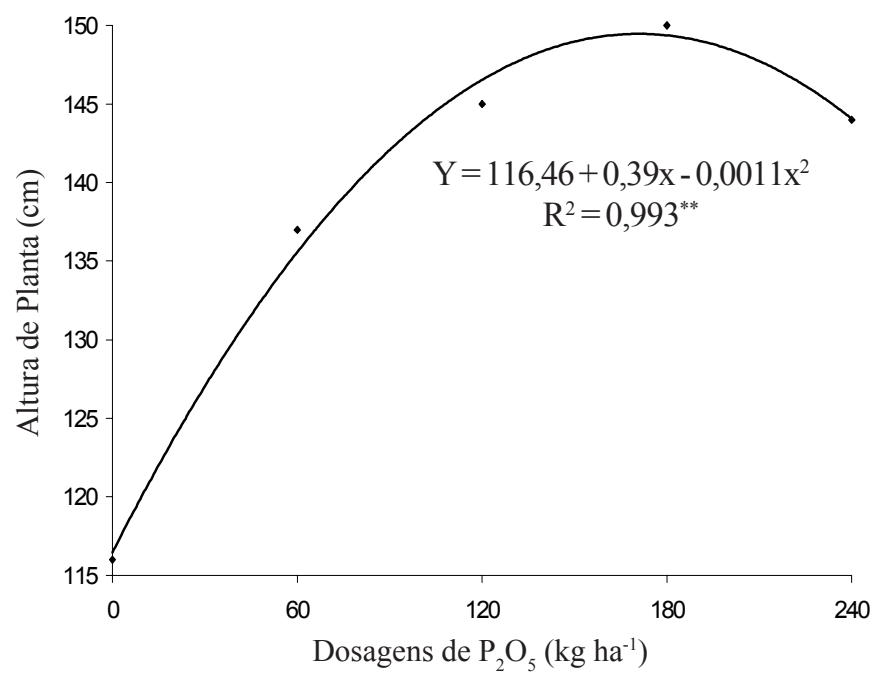

Figura 2. Altura de planta de milho, cv. BR5033, em função de dosagens crescentes de $\mathrm{P}$ aplicadas ao solo

A curva de resposta da variação do rendimento da cultura em função das dosagens crescentes de $\mathrm{N}$ aplicadas ao solo (Figura 3), apresentou efeito quadrático altamente significativo $(p \leq 0,01)$ em que, de acordo com a função de produção obtida, o rendimento máximo de grãos $\left(2.257,2 \mathrm{~kg} \mathrm{ha}^{-1}\right)$ seria teoricamente atingido com aplicação de 111,1 kg ha-1 de N; comportamento similar foi obtido por Santos (1997) na mesma região, com o milho pipoca (Zea mays everta) quando, testando as dosagens de nitrogênio $0,30,60$ e $120 \mathrm{~kg} \mathrm{ha}^{-1} \mathrm{de} \mathrm{N}$, observou que o máximo rendimento da cultura, $3.556 \mathrm{~kg} \mathrm{ha}^{-1}$ de grãos foi alcançado com a dosagem de $60 \mathrm{~kg} \mathrm{ha}^{-1}$ de N, porém para outras localidades do país Melgar et al. (1991) constataram que o rendimento do milho se comportou de forma linear, em função das dosagens de nitrogênio $\left(40,80\right.$ e $120 \mathrm{~kg} \mathrm{ha}^{-1}$ de N) aplicadas ao solo. Para Cantarella (1993) a magnitude das respostas ao nitrogênio em ensaios conduzidos no Brasil, tem sido variável, mas a maioria dos estudos indica respostas significativas a dosagens entre 30 e $90 \mathrm{~kg} \mathrm{ha}^{-1}$ de N, devido, em parte, aos níveis de produtividade relativamente baixos. Almeida et al. (1985) afirmam que, em várias localidades, a dosagem de $85 \mathrm{~kg} \mathrm{ha}^{-1}$ de $\mathrm{N}$ foi responsável pela obtenção de uma produtividade máxima econômica de $3.705 \mathrm{~kg} \mathrm{ha}^{-1}$ de grãos de milho. 


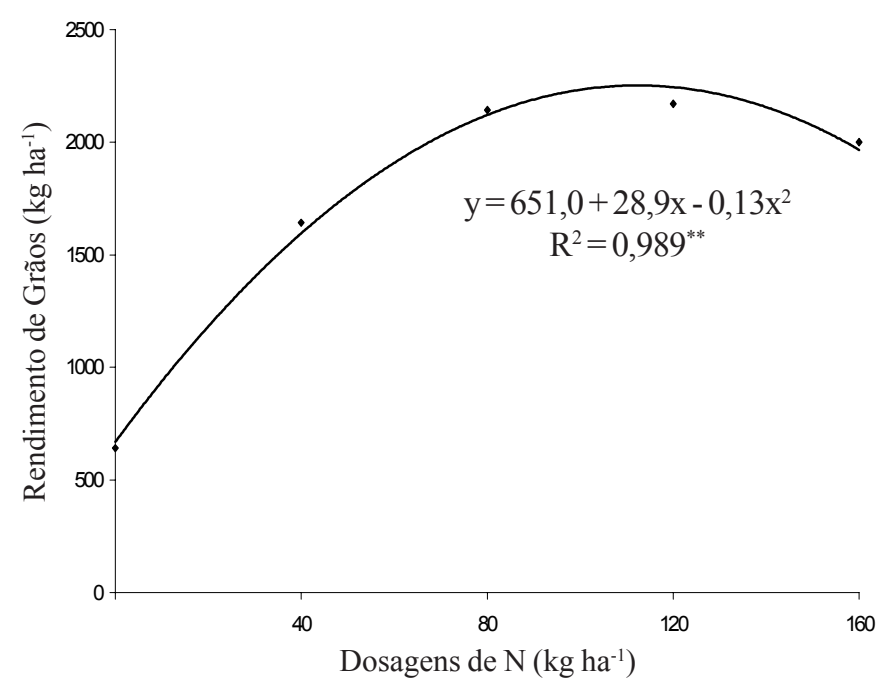

Figura 3. Curva de resposta do rendimento de grãos de milho, cv. BR5033, em função de dosagens crescentes de $\mathrm{N}$ aplicadas ao solo

A Figura 4 representa a variação do rendimento do milho em função das dosagens de $\mathrm{P}_{2} \mathrm{O}_{5}$ aplicadas ao solo. Verifica-se que o rendimento da cultura aumentou com o aumento das dosagens de $\mathrm{P}_{2} \mathrm{O}_{5}$, até o nível de $197,6 \mathrm{~kg} \mathrm{ha}^{-1}$ cuja função atinge um ponto de máxima (2.258,2 $\mathrm{kg} \mathrm{ha}^{-1}$ de grãos) para, então, apresentar tendência contínua decrescente. Respostas do milho ao fósforo também são referenciadas nos trabalhos de Oliveira et al. (1982) e Lobato (1982) para várias localidades do país.

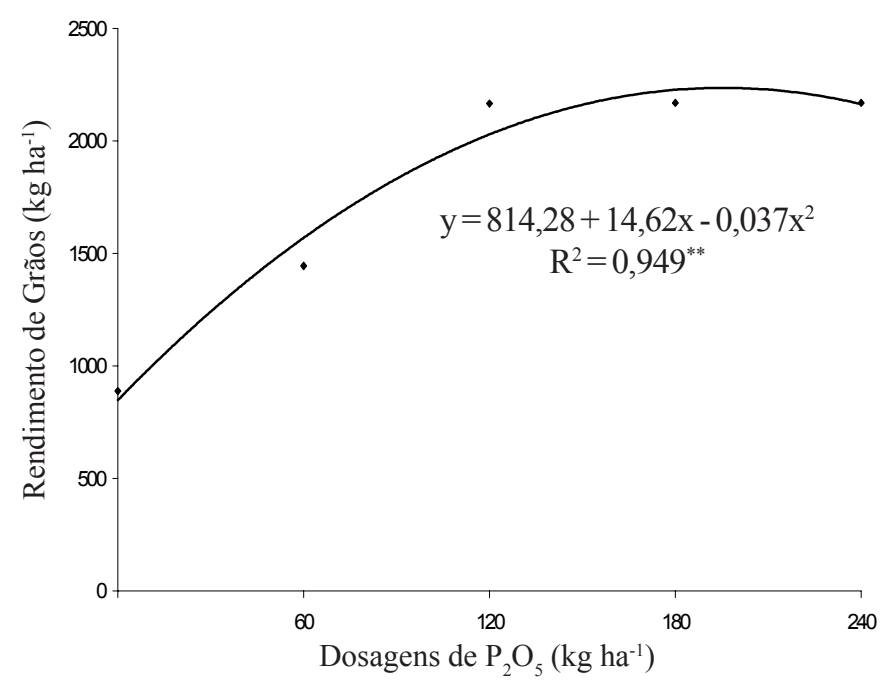

Figura 4. Curva de resposta do rendimento de grãos de milho, cv. BR5033, em função de dosagens crescentes de P aplicadas ao solo

\section{CONCLUSÕES}

1. As variáveis analisadas apresentaram respostas positivas e significativas à aplicação de nitrogênio e fósforo.

2. As aplicações de $111,1 \mathrm{~kg} \mathrm{ha}^{-1}$ de nitrogênio e de $197,6 \mathrm{~kg} \mathrm{ha}^{-1}$ de fósforo $\left(\mathrm{P}_{2} \mathrm{O}_{5}\right)$ propiciaram as maiores produtividades de milho.

\section{REFERÊNCIAS BIBLIOGRÁFICAS}

ALMEIDA, D.L. de; DE-POLLI, H.; PESSANHA, G.G.; AMARAL SOBRINHO, N.M.B. Adubação nitrogenada nos Estados de Rio de Janeiro e Espírito Santo. In: REUNIÃO BRASILEIRA DE FERTILIDADE DO SOLO, 16, Ilhéus, 1985. Anais... Ilhéus, BA: CEPLAC/SBCS, 1985, p.81-106.

BRAGA, J.M. Avaliação da fertilidade do solo (Ensaio de campo). Viçosa, MG: UFV. Imp. Universitária, 1992. 101p.

BRASIL. Ministério da Agricultura. Levantamento exploratório: Reconhecimento de solos do Estado da Paraíba. Rio de Janeiro: MA/CONTAP/USAID/SUDENE, 1972. 670p. Boletim Técnico 15

CANTARELLA, H. Calagem e adubação do milho. In: SIMPÓSIO SOBRE FATORES QUE AFETAM A PRODUTIVIDADE DO MILHO E SORGO, Vitória, ES, 1990. Piracicaba: POTAFOS, 1993.p.147-196.

CANTARELLA, H.; RAIJ, B. van. Adubação nitrogenada no Estado de São Paulo. In: SANTANA, M.B.M. Adubação nitrogenada no Brasil. Ilhéus: CEPLAC, Sociedade Brasileira de Ciência do Solo, p.47-49. 1986

CARVALHO, H.W.L. de; SANTOS, M.X. dos; LEAL, M. de L. da S.; CARDOSO, M.J. Variedades de milho para o Nordeste Brasileiro. EMBRAPA - CPATC. maio 1997. 1p, Comunicado Técnico

FORMASIERE FILHO, D. A cultura do milho. São Paulo: FUNEP, 1992. p.33-40.

FRANÇA, G.E. de; BAHIA FILHO, A.F.C.; VASCONCELOS, C.A.; SANTOS, H.L. Adubação no Estado de Minas Gerais. In: SANTANA, M.B.M. (Coord.). Adubação nitrogenada no Brasil. Ilhéus: CEPLAC, Sociedade Brasileira de Ciência do Solo, p.107-124. 1986.

GAMBOA, A. La fertilizacion del maiz. Berna: Instituto Internacional de la Potassa, p.72. 1980. Boletim, 2

LOBATO, E. Adubação fosfatada em solos da região CentroOeste. In: OLIVERIA, A.J. de (ed.). Adubação fosfatada no Brasil. Brasília, DF: EMBRAPA-DID, 1982.326p.

MELGAR, R J.; SMYTH, T.J.; CRAVO, M.S.; SÁNCHEZ, P.A. Doses e épocas de aplicação de fertilizantes nitrogenados para o milho em Latossolo da Amazônia Central. Revista Brasileira de Ciência do Solo. Campinas, v.15, p.196-289. 1991.

MUZILLI, O. Adubação fosfatada no Estado do Paraná. In: Oliveira, A.J. ed. Adubação fosfatada no Brasil. Brasília: EMBRAPA, 1982.p.61-100.

MUZILLI, O.; OLIVEIRA, E.L. de; CALEGARI, A. Adubação do milho. Campinas: Cargill, v.4, 1989, 28p.

OLIVEIRA, A.J.; LOURENÇO, S.; GOEDERT, W.J. Adubação fosfatada no Brasil. Brasília: EMBRAPA, 1982. 326p.

SANTOS, A.C. Espaçamento x níveis de nitrogênio. Efeito sobre a produção e o desenvolvimento de duas cultivares de milho pipoca (Zea mays everta). Areia, PB, 1997. 73p. Monografia Graduação

VIEIRA, J.M.; FONTES, L.A.; GALVÃO, J.D. Produção de grãos, teores de proteínas e de lisina em cultivares de milho Opaco-2 e normal, em diferentes níveis de adubação nitrogenada e fosfatada. Experimento UFV, Viçosa v.21, n3, p.49-69.1976.

VIOLA, E.A. Considerações sobre a cultura do milho. In: Instituto de Pesquisa Agronômica. IPAGRO, Porto Alegre n.23 p.3-8, 1980. 